\title{
Properties of a deep seismic waveguide measured with an optical fiber
}

\author{
Ariel Lellouch $\odot,{ }^{*}$ Ettore Biondi $\odot$, and Biondo L. Biondi $\odot$ \\ Stanford University, Stanford, California 94035, USA \\ Bin Luo $\odot$ and Ge Jin $\odot$ \\ Colorado School of Mines, Golden, Colorado 80401, USA \\ Mark A. Meadows \\ Formerly Chevron Energy Technology Company, San Ramon, California 94583, USA
}

(Received 15 September 2020; accepted 4 February 2021; published 19 February 2021)

\begin{abstract}
Low-velocity zones located deep in the subsurface can act as seismic waveguides. Traditionally, their experimental observation has been limited by the practical challenges of in situ recording. We use a measurement technique in which optical fibers are turned into seismic sensors. The fiber is deployed along a horizontal well drilled inside a 15-m-thin shale formation at a depth of about $2 \mathrm{~km}$. Owing to the high-resolution recording of the optical fiber, we can distinctly observe three previously elusive guided wave modes over a wide frequency range. As their propagation is primarily confined to the waveguide and strongly depends on its seismic properties, such guided waves hold tremendous potential for high-resolution imaging of deep low-velocity structures, such as fault zones, saline aquifers, and hydrocarbon reservoirs.
\end{abstract}

DOI: 10.1103/PhysRevResearch.3.013164

\section{INTRODUCTION}

Waveguides are structures in which waves propagate with minimal loss. This property is the result of confining wave propagation to within the spatial extent of the waveguide. It can be electromagnetic, acoustic, or elastic. For example, optical fibers and transmission cables are man-made electromagnetic waveguides. The atmospheric duct can guide radio and light waves over the horizon. The sound fixing and ranging (SOFAR) ocean channel is an acoustic waveguide through which whales communicate at long distances [1]. Ultrasonic guided waves are often used in nondestructive testing of pipes [2] and structures [3].

In seismology, guided waves are the result of boundary conditions imposed on the seismic wave equation [4]. The most commonly encountered conditions include a free surface, an interface between two layers, and an effectively infinite half space. Different combinations of these boundary conditions give rise to a variety of possible guided-wave modes, each with different properties [5]. For example, Love waves, which are a constructive interference of horizontally polarized SH waves, arise from the combination of a freesurface condition and a solid low-velocity layer overlying a faster infinite half space [6]. The same boundary conditions can also sustain guided waves with particle motion in the

\footnotetext{
*Corresponding author: ariellel@ stanford.edu

Published by the American Physical Society under the terms of the Creative Commons Attribution 4.0 International license. Further distribution of this work must maintain attribution to the author(s) and the published article's title, journal citation, and DOI.
}

vertical plane. These may be either normal (SV waves) or leaky (P waves) modes [7]. A crustal waveguide may also occur if the low-velocity layer is a fluid [8].

In this paper, we focus on different boundary conditions. A low-velocity zone is embedded between two different high-velocity layers. The fast layers can be either infinite half spaces, in which case the simplest waveguide arises, or themselves be bounded by other layers. Guided waves propagating in low-velocity structures have been studied in fault zones [9-14], coal seams [15-17], and cross-well surveys in exploration seismology $[18,19]$. Guided waves are primarily confined to the low-velocity zone in which they propagate. For deep, narrow waveguides, sensors deployed at the Earth's surface will only record faint signals emanating from short-wavelength guided waves. Instead, downhole sensors positioned in or close to the waveguide are required to properly observe them. Deploying such sensors is challenging, costly, and might be effectively limited by the harsh pressure and temperature conditions in deep waveguides. In addition, sensor spacing dictates the spatial aliasing boundary conditions. Wavelengths shorter than half of the sensor spacing cannot be properly recorded, thus limiting our highfrequency understanding of the waveguide properties. As a result, previous studies of deep waveguides have shown restricted field-data recording that does not allow for unequivocal analysis of guided-wave properties.

Distributed acoustic sensing (DAS), a new seismic sensing technology, helps to alleviate acquisition limitations. By turning an optical fiber into a seismic sensor through an appropriate optical apparatus called an interrogator, DAS allows for unaliased spatial sampling, even at high frequencies [20,21]. Typically, the distance between adjacent measurement points is $1-10 \mathrm{~m}$, with over 1000 samples per second. DAS measures 
(a)

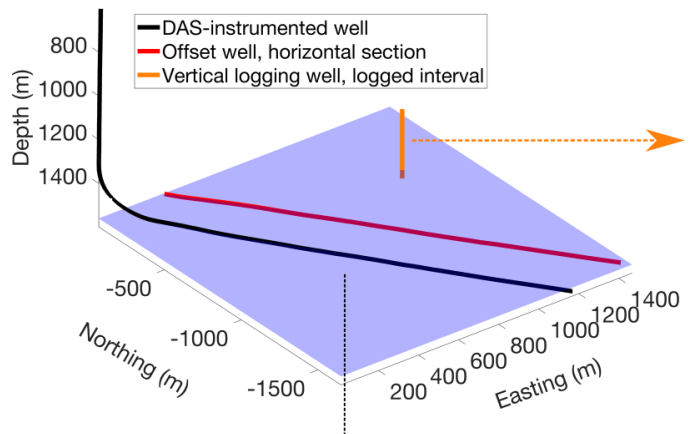

(b)

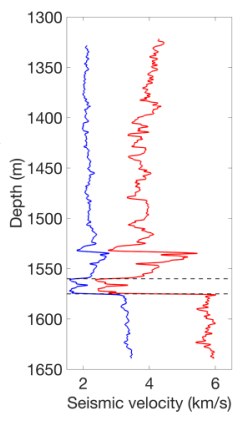

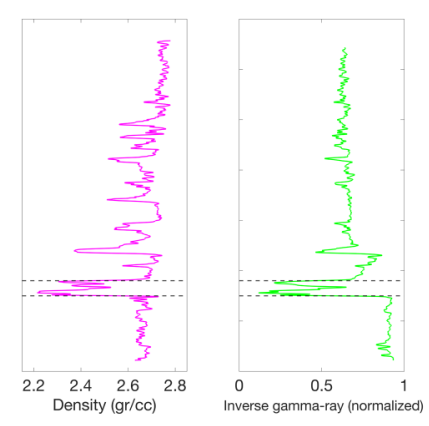

(c)

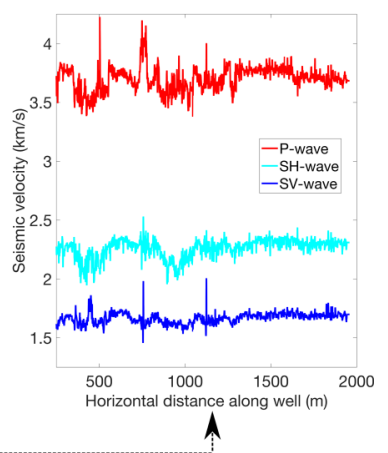

FIG. 1. (a) Experimental setup showing the fiber-instrumented well (black) and the offset well (red). Both wells are perforated. (b) Logging results of a vertical well (schematically marked in orange) including P (red) and S (blue) velocities, density (pink), and inverse of the shale content estimated by gamma rays. The 15-m-thick reservoir boundaries are denoted by dashed black lines. (c) Seismic velocity logging conducted in the horizontal section of the DAS-instrumented (black) well. P-wave (red), SH-wave (cyan), and SV-wave (blue) velocities are shown. Shear-wave splitting due to anisotropy is evident, and the P-wave velocity is much higher than that measured by the vertical log in the reservoir interval.

the directional strain parallel to the fiber but is not a point measurement. Instead, it records the total strain over a distance called the gauge length, which is usually about $10 \mathrm{~m}$. Up to tens of $\mathrm{km}$ of fiber can be simultaneously recorded with a single interrogator $[22,23]$. The DAS fiber can be permanently installed in any cased well, vertical or deviated, and is resistant to harsh environmental conditions. When it is installed outside the casing, it does not prohibit nor suffers strong noise from other operations in the well.

We study a deep seismic waveguide in which a DAS fiber was deployed. The low-velocity zone is an unconventional hydrocarbon shale reservoir located in a major basin in the continental United States of America. It lies at a depth of almost $2 \mathrm{~km}$ from the surface and is bounded between stiffer, higher-velocity rocks. Its thickness is approximately $15 \mathrm{~m}$ in the study area. The datum used in this study is shifted in depth by about $400 \mathrm{~m}$ from the wellhead elevation. The layered shale structure induces strong vertically transverse isotropy (VTI) $[24,25]$. We show in situ measurements of the dispersive properties of guided waves over a wide frequency range $(50-700 \mathrm{~Hz})$ and propagation distances approaching $1 \mathrm{~km}$. We complement our interpretation with semianalytical solutions and wave-equation modeling.

\section{DATA ACQUISITION}

Figure 1 summarizes the experimental design and well logs acquired in the area. The DAS fiber was deployed along a deviated well drilled into an unconventional shale reservoir. The horizontal section of the same well was perforated preceding production. Perforation shots were oriented $180^{\circ}$ away from the fiber so as to not damage it, and 97 of those were recorded by the DAS array [26]. Forty-three perforations from a parallel horizontal well, located in the shale reservoir approximately 260-280 m away from the fiber-instrumented well, were also recorded. Detailed logging was conducted in a vertical well, drilled deeper than the reservoir, and located several hundred meters away from the two horizontal wells. The vertical log shows that the reservoir, whose high shale content is corrobo- rated by the gamma-ray log, has significantly lower velocities and density than its surroundings. In addition to the vertical $\log$, the horizontal section of the fiber-instrumented well was also logged. The difference in P-wave velocity between the vertical measurement in the reservoir interval and the horizontal log indicates strong anisotropy, and so does the shear-wave splitting measured in the horizontal S-wave log.

\section{GUIDED WAVE PROPERTIES}

\section{A. Numerical modeling}

The elastic structure of the waveguide can be simplified to a three-layer model, with the top and bottom acting as effectively infinite half spaces. We conduct an illustrative twodimensional (2D) isotropic wave-equation modeling exercise with constant density. The vertically oriented force source is located within the low-velocity reservoir. Figure 2 shows snapshots of the particle motion in the horizontal direction, as well as the seismic velocities of the three layers. As the modeling is $2 \mathrm{D}$, the $\mathrm{SH}$ modes are not present. However, two distinct modes of guided waves, confined to the low-velocity reservoir, are visible. They are the leaky and P-SV normal modes, and both are clearly dispersive, with low frequencies propagating faster than high frequencies. The P-SV normal mode propagates more slowly than the leaky mode, and decays exponentially away from the boundaries outside the reservoir. However, this decay is wavelength dependent, and thus the skin-depth penetration extends farther for the lower frequencies. The leaky mode, in contrast, radiates body-wave energy into the surrounding formation in the form of $\mathrm{S}$ waves. It is thus not a pure guided wave. It can be viewed as a constructive interference of $\mathrm{P}$-wave reflections from the reservoir interfaces. However, at each reverberation, some energy is lost to a P-to-S conversion.

One of the most noticeable features of guided waves is their dispersive nature. As a kinematic property, it is strongly linked to the elastic structure and almost unaffected by instrumentation type. Therefore, we focus on analyzing dispersion curves, which represent the phase velocities of different propagating 




FIG. 2. Snapshots of the horizontal component of wave propagation in a seismic waveguide. Images are taken $0.1 \mathrm{~s}$ apart. The seismic velocities of the three layers are marked in the top snapshot. Blue/orange: Propagation above the waveguide. Black/yellow: In the waveguide. Pink/green: Below the waveguide. Two distinct types of waves confined to the low-velocity reservoir (marked in dotted black lines) are visible: Leaky (red arrow) and P-SV normal (purple arrow) modes. For the leaky modes, there is a strong $\mathrm{S}$-wave radiation into the layers above and below the reservoir (dotted red arrows). The normal modes decay exponentially away from the boundaries outside the reservoir, with a wavelength-dependent skin depth.

frequencies [27]. Figure 3(a) shows the dispersive behavior of modeled seismic data extracted along a receiver line located $5 \mathrm{~m}$ above the bottom of the low-velocity layer. The mapping from the space-time domain to the frequency-phase velocity domain is described in Ref. [27]. The normal P-SV guided waves consist of six different modes in the frequency range we study. At a given frequency, the phase velocity increases with mode number, with the fundamental mode being the slowest.

\section{B. Semianalytical solutions}

While wave-equation modeling yields the full seismic wave field in more complicated structures, it is not always straightforward to separate the guided waves from other seismic phases. In addition, it is computationally expensive, and, for the frequency range of interest, modeling 3D wave propagation can be prohibitive. An alternative approach to computing dispersion curves, which can be used for layered structures, is a semianalytical solution based on the propagator matrix technique [28-32]. This approach is widely applied for boundary-wave dispersion computation in multilayered media. The propagator matrix method can be semianalytically derived from the equations of motion and Hooke's law, and is represented as a generic form of the dispersion equation [33], formulated as $\operatorname{det}\left(U^{T} P V\right)=0$, where $P$ is the product of $n$ propagator matrices of layers in a $1 \mathrm{D} n$-layered model, and $U$ and $V$ are the boundary condition matrices at the top and base of the entire model, respectively. The explicit form of the dispersion equation is determined by the given 1D multilayered structure and the boundary conditions at the top and base interfaces, whereas the solution of the dispersion equation yields the multimodal curves for guided-wave dispersion prediction. Different types of boundary waves stem from different boundary conditions, which require different representations of the

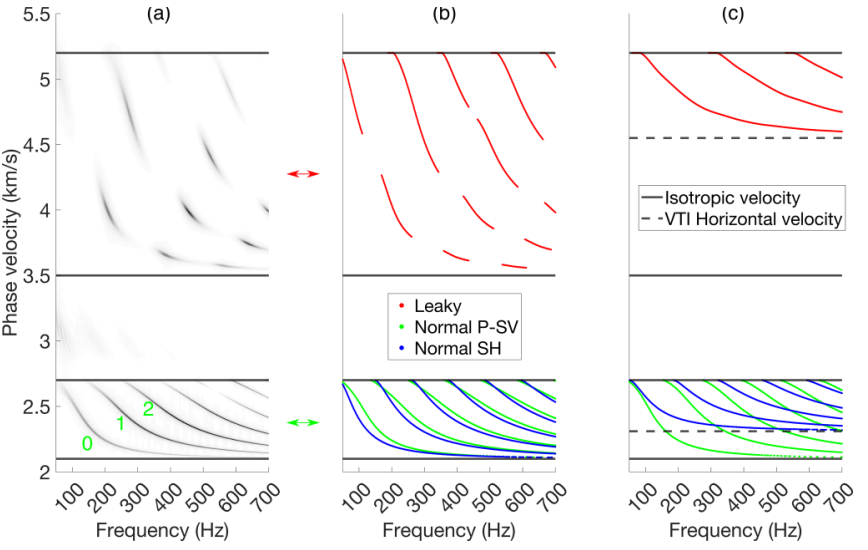

FIG. 3. Guided-wave dispersion curves in a three-layer model. (a) Results of $2 \mathrm{D}$ isotropic wave-equation modeling. P-SV modes (pointed by green arrow) and leaky modes (pointed by red arrow) are visible. For both, there are multiple propagation modes. They are marked as 0 (fundamental), 1, and 2 for normal P-SV waves. (b) Analytical solution for the 3D isotropic case. The leaky $\mathrm{P}$ waves (red) have a discontinuous spectrum. Both leaky and normal P-SV modes (green) match the wave-equation solution shown in (a). The normal SH mode (blue) differs from the P-SV case. (c) Analytical solution for the 3D case with an anisotropic low-velocity layer. Normal P-SV modes are almost unchanged and are still bounded by the S-wave velocity in the vertical direction. SH modes are bounded by the horizontal velocity of the layer, which is faster. Leaky modes are bounded by the $\mathrm{P}$-wave velocity in the horizontal direction, which is faster than the vertical; as a result, only three continuous modes are present.

matrices $U$ and $V$. For deep guided waves, the waveguide can assumed to be bounded by high-velocity infinite half spaces that require both $U$ and $V$ to represent a no-energy-influx boundary condition and a continuity of displacement and traction across the interface. For both isotropic and VTI cases, the dispersion equation decouples into two independent equations that predict guided P-SV and guided SH-wave dispersion characteristics, respectively $[31,34,35]$. For a layered structure with known material properties and layer thicknesses, the term $\operatorname{det}\left(U^{T} P V\right)=0$ describes a multivariate matrix determinant function in the phase velocity-frequency domain, in which the zeros are modal solutions to the dispersion equation. We perform a grid search of the matrix determinant function in the desired phase velocity-frequency domain and find the zeros by automatically picking each local minimum below a threshold of 0.001 in the resulting determinant $D$. This approach computes all possible guided-wave modes while being agnostic to the seismic source. Therefore, it does not predict the excitation and relative strength of the different modes. Nonetheless, it can quickly compute solutions for 3D propagation in layered media, both isotropic and VTI, over a wide frequency range. Figure 3(b) shows perfect agreement between P-SV normal and leaky modes predicted by the semianalytical approach and those generated by wave-equation modeling. The propagatormatrix method also predicts the $\mathrm{SH}$ normal modes, which cannot be modeled with a 2D wave equation. SH-mode properties differ from those of P-SV normal modes despite having the same phase-velocity boundaries. As shown in Fig. 3, 
these boundaries are given by the shear-wave velocities of the reservoir $(2.1 \mathrm{~km} / \mathrm{s})$ and of the layer above it $(2.7 \mathrm{~km} / \mathrm{s})$, which is slower than the layer below $(3.3 \mathrm{~km} / \mathrm{s})$. Interestingly, the leaky-mode spectrum is discontinuous in both the wave-equation and semianalytical cases, indicating that, for the given structure, such a feature is an intrinsic property of guided-mode propagation. The phase-velocity boundaries of the leaky modes are given by the P-wave velocities of the reservoir $(3.5 \mathrm{~km} / \mathrm{s})$ and of the layer above it $(5.2 \mathrm{~km} / \mathrm{s})$.

We use the same propagator-matrix method to compute dispersion curves in the presence of anisotropy. In our model, the vertical velocities are the same as for the isotropic case. The shear-wave velocity along the horizontal direction is higher than the vertical velocity by $10 \%$, and the P-wave horizontal-to-vertical velocity difference is $30 \%$. The VTI $\mathrm{P}$-wave velocity at $45^{\circ}$ from the vertical is higher by $15 \%$ than the vertical velocity. Dispersion curves shown in Fig. 3(c) are very different from those shown in Fig. 3(b). The leaky waves are bounded by the horizontal P-wave velocity $(4.55 \mathrm{~km} / \mathrm{s})$, fewer modes are visible, and their spectra are continuous. For the normal modes, the P-SV are only slightly changed, and are still bounded by the slower vertical S-wave velocity of the reservoir. Normal SH modes, however, are bounded by the horizontal S-wave velocity $(2.32 \mathrm{~km} / \mathrm{s})$, and a clear separation from the P-SV modes is visible.

\section{FIELD OBSERVATIONS}

In Fig. 4, we show a summary of observed field data. The frequency content, which extends up to $700 \mathrm{~Hz}$, is remarkable for seismic applications. Whether the source is generated along the DAS-instrumented well or in an offset well influences which modes are excited. The source focal mechanism, whose analysis is outside the scope of this paper, has a strong effect as well. For a source along the recording well, leaky waves and normal P-SV modes dominate the record, whereas the normal SH mode is weak, as expected. As DAS measures strain along the fiber, there is very little sensitivity to the out-of-plane particle motion induced by $\mathrm{SH}$ waves. For the cross-well case, however, SH waves are expected to carry most of the strain energy in this recording geometry, and they are indeed much stronger than the P-SV waves. There is an obvious velocity difference between the two normal modes, as expected by the anisotropy measured along the reservoir, and as demonstrated in the semianalytical examples shown earlier. Their asymptotic velocities, $2.3 \mathrm{~km} / \mathrm{s}(\mathrm{SH})$ and $1.7 \mathrm{~km} / \mathrm{s}$ (P-SV), match the log velocities acquired in the reservoir and shown in Fig. 1. For the leaky waves, the $3.8 \mathrm{~km} / \mathrm{s}$ asymptote matches the horizontal (fast) P-wave velocity. For the leaky and normal SH modes, there are two distinct branches with different dispersive characteristics. Despite many modeling tests, we were not able to recreate them using the seismic velocities logged in a vertical well located several hundred meters away from the study area. Structural inversion is outside the scope of this study, but from initial tests it appears that the two branches lie too close together to represent a fundamental and first mode from the same waveguide. The most plausible explanation for this pattern is the presence of two separate waveguides with slightly different properties. In this case, we are observing two different fundamental modes (a)

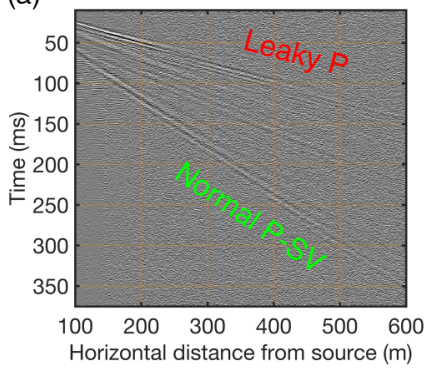

(c)

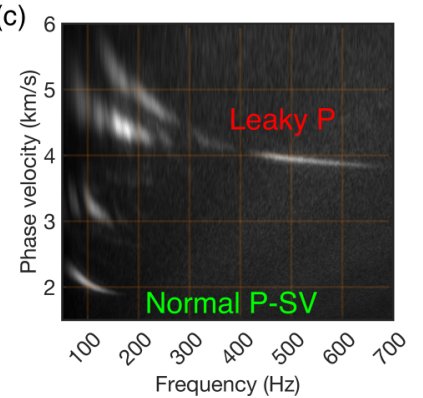

(b)

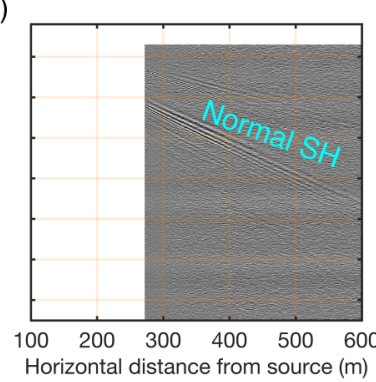

(d)

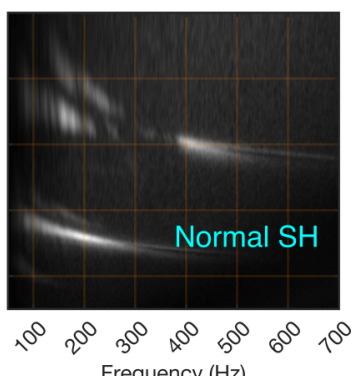

FIG. 4. Field data observations. We show time-domain records of a single perforation shot along the DAS-instrumented well (a) and in the offset well (b), after mapping to horizontal source-channel distance. Leaky (red), normal SH (cyan), and normal P-SV (green) modes can be observed. Dispersion images obtained by summation of 20 sources along the DAS-instrumented well (c) and 43 sources in the offset well (d) show the dispersive properties of the different modes. There are two main leaky branches (red) in which the spectrum is discontinuous, two normal SH branches (blue), and a single normal P-SV branch.

propagating in different low-velocity structures. The two branches appear in data from both the fiber-instrumented and offset wells, and we thus suspect that a continuous structure exists between these two wells that does not extend to the vertical well where the logs were acquired. Possible evidence for this structure is the high-velocity, low-shale-content intrusion that appears around the middle of the reservoir (Fig. 1), whose presence could potentially split the reservoir into two different waveguides.

\section{CONCLUSIONS AND OUTLOOK}

Perforation shots excite guided waves that propagate long distances with minimal losses. They undergo cylindrical propagation, which is essentially a $2.5 \mathrm{D}$ phenomenon, and are primarily confined to the low-velocity waveguide. When generated by a high-frequency source, they can travel for hundreds of meters with a frequency content as high as $700 \mathrm{~Hz}$. Their wavelengths, in this case, are only several meters, significantly less than the reservoir thickness. From a practical point of view, they can be recorded without aliasing only with a DAS array. We focus on their kinematic properties in the form of dispersion curves and give a high-resolution observation of their properties. We also confirm the accuracy of computational tools that can predict their behavior for a given anisotropic structure.

Guided waves hold tremendous potential for highresolution imaging and reconstruction of low-velocity 
subsurface structures, such as fault zones, saline aquifers, and hydrocarbon reservoirs. They are highly sensitive to elastic properties, including anisotropy, and can be used to complement surface-based seismic and well logs. DAS directivity plays a major role in their response and can partly explain the relative strength of the different modes. For a more detailed amplitude analysis, however, both the source focal mechanism and behavior of the guided waves at different depths within the waveguide need to be taken into account in addition to the kinematic properties.

\section{ACKNOWLEDGMENTS}

We are thankful to Chevron ETC for providing us field data and permission to publish. The SEP affiliate program partially supported A.L., E.B., and B.L.B. A.L. was also supported by the Israeli Ministry of Energy under the program for postdoctoral studies in leading universities and the CoRE project supported by Chevron. B.L. was supported by the Reservoir Characterization Project (RCP) affiliate program. The waveequation modeling code was codeveloped with Guillaume Barnier (SEP).
[1] R. Payne and D. Webb, Orientation by means of long range acoustic signaling in baleen whales, Ann. N.Y. Acad. Sci. 188, 110 (1971)

[2] M. J. Lowe, D. N. Alleyne, and P. Cawley, Defect detection in pipes using guided waves, Ultrasonics 36, 147 (1998).

[3] P. Cawley and D. Alleyne, The use of Lamb waves for the long range inspection of large structures, Ultrasonics 34, 287 (1996).

[4] K. Aki and P. G. Richards, Quantitative Seismology (University Science Books, Mill Valley, CA, 2002), p. 704.

[5] R. E. Sheriff and L. P. Geldart, Exploration Seismology (Cambridge University Press, Cambridge, U.K., 1995), pp. 483-486.

[6] A. E. H. Love, Some Problems of Geodynamics (Cambridge University Press, Cambridge, U.K., 1911).

[7] F. Gilbert, Propagation of transient leaking modes in a stratified elastic waveguide, Rev. Geophys. 2, 123 (1964).

[8] R. A. Phinney, Leaking modes in the crustal waveguide: 1 . The oceanic PL wave, J. Geophys. Res. 66, 1445 (1961).

[9] Y. Ben-Zion, Properties of seismic fault zone waves and their utility for imaging low-velocity structures, J. Geophys. Res. 103, 12567 (1998).

[10] Z. Peng, Y. Ben-Zion, A. J. Michael, and L. Zhu, Quantitative analysis of seismic fault zone waves in the rupture zone of the 1992 Landers, California, earthquake: Evidence for a shallow trapping structure, Geophys. J. Int. 155, 1021 (2003).

[11] V. A. Korneev, R. M. Nadeau, and T. V. McEvilly, Seismological studies at Parkfield IX: Fault-zone imaging using guided wave attenuation, Bull. Seismol. Soc. Am. 93, 1415 (2003).

[12] Y.-G. Li, K. Aki, D. Adams, A. Hasemi, and W. H. K. Lee, Seismic guided waves trapped in the fault zone of the Landers, California, earthquake of 1992, J. Geophys. Res. 99, 11705 (1994).

[13] W. L. Ellsworth and P. E. Malin, Deep rock damage in the San Andreas Fault revealed by P- and S-type fault-zone-guided waves, Geol. Soc. Spec. Publ. 359, 39 (2011).

[14] H. Igel, G. Jahnke, and Y. Ben-Zion, Numerical simulation of fault zone guided waves: Accuracy and 3-D effects, Pure Appl. Geophys. 159, 2067 (2002).

[15] F. Evison, A coal seam as a guide for seismic energy, Nature (London) 176, 1224 (1955).

[16] T. C. Krey, Channel waves as a tool of applied geophysics in coal mining, Geophysics 28, 701 (1963).

[17] D. J. Buchanan, The propagation of attenuated SH channel waves, Geophys. Prospect. 26, 16 (1976).

[18] C. E. Krohn, Cross-well continuity logging using guided seismic waves, Leading Edge 11, 39 (1992).

[19] M. Lou and S. Crampin, Dispersion of guided waves in thin anisotropic waveguides, Geophys. J. Int. 107, 545 (1991).
[20] P. Jousset, T. Reinsch, T. Ryberg, H. Blanck, A. Clarke, R. Aghayev, G. P. Hersir, J. Henninges, M. Weber, and C. M. Krawczyk, Dynamic strain determination using fibre-optic cables allows imaging of seismological and structural features, Nat. Commun. 9, 2509 (2018).

[21] T. Parker, S. Shatalin, and M. Farhadiroushan, Distributed Acoustic Sensing - A new tool for seismic applications, First Break 32, 61 (2014).

[22] N. J. Lindsey, T. Craig Dawe, and J. B. Ajo-Franklin, Illuminating seafloor faults and ocean dynamics with dark fiber distributed acoustic sensing, Science 366, 1103 (2019).

[23] J. B. Ajo-Franklin, S. Dou, N. J. Lindsey, I. Monga, C. Tracy, M. Robertson, V. Rodriguez Tribaldos, C. Ulrich, B. Freifeld, T. Daley, and X. Li, Distributed acoustic sensing using dark fiber for near-surface characterization and broadband seismic event detection, Sci. Rep. 9, 1328 (2019).

[24] L. Thomsen, Weak elastic anisotropy, Geophysics 51, 1954 (1986).

[25] C. M. Sayers, Seismic anisotropy of shales, Geophys. Prospect. 53, 667 (2005).

[26] A. Lellouch, S. Horne, M. A. Meadows, S. Farris, T. Nemeth, and B. Biondi, DAS observation of guided waves in a shale reservoir generated by perforation shots, Leading Edge 38, 858 (2019).

[27] C. B. Park, R. D. Miller, and J. Xia, Multichannel analysis of surface waves, Geophysics 64, 800 (1999).

[28] W. T. Thomson, Transmission of elastic waves through a stratified solid medium, J. Appl. Phys. 21, 89 (1950).

[29] N. A. Haskell, The dispersion of surface waves on multilayered media, Bull. Seismol. Soc. Am. 43, 17 (1953).

[30] F. Gilbert and G. E. Backus, Propagator matrices in elastic wave and vibration problems, Geophysics 31, 326 (1966).

[31] J. W. Dunkin, Computation of modal solutions in layered, elastic media at high frequencies, Bull. Seismol. Soc. Am. 55, 335 (1965).

[32] C. Y. Wang and R. B. Herrmann, A numerical study of P-, $\mathrm{SV}-$, and SH-wave generation in a plane layered medium, Bull. Seismol. Soc. Am. 70, 1015 (1980).

[33] P. W. Buchen and R. Ben-Hador, Free-mode surface-wave computations, Geophys. J. Int. 124, 869 (1996).

[34] T. Liu, C. Zhao, and Y. Duan, Generalized transfer matrix method for propagation of surface waves in layered azimuthally anisotropic half-space, Geophys. J. Int. 190, 1204 (2012).

[35] T. Ikeda and T. Matsuoka, Computation of Rayleigh waves on transversely isotropic media by the reduced delta matrix method, Bull. Seismol. Soc. Am. 103, 2083 (2013). 